\title{
Modeling of Biomass Production of Haematococcus pluvialis
}

\author{
Rosana M. Galvão ${ }^{1}$, Tarlen S. Santana ${ }^{1}$, Cristiano H. O. Fontes ${ }^{2}$, Emerson A. Sales ${ }^{1,2}$ \\ ${ }^{1}$ Laboratory of Bioenergy and Catalysis (LABEC), Polytechnic School, Federal University of Bahia, Salvador-Bahia, Brazil \\ ${ }^{2}$ Post-Graduate Program in Industrial Engineering (PEI), Polytechnic School, Federal University of Bahia, \\ Salvador-Bahia, Brazil \\ Email: rosamgalvao@gmail.com, tarlen_santana@hotmail.com,cfontes@ufba.br, eas@ufba.br
}

Received May 9, 2013; revised June 9, 2013; accepted June 16, 2013

Copyright (C) 2013 Rosana M. Galvão et al. This is an open access article distributed under the Creative Commons Attribution License, which permits unrestricted use, distribution, and reproduction in any medium, provided the original work is properly cited.

\begin{abstract}
Microalgae cultivation is justified by the production of high-value fine chemicals and biofuels, essential to reduce the emissions of gases that cause global warming. This paper presents a study of the growth of microalgae Haematococcus pluvialis considering light conditions from 2000 to 10,000 lux, temperature $22^{\circ} \mathrm{C}$ and $\mathrm{pH}$ in the $6.5-12.5$ range. The experiments were performed in 4 liter flat plate photobioreactors using the Rudic culture medium. The biomass growth was measured by counting cells in a Neubauer chamber. Both the light intensity and the $\mathrm{pH}$ of the medium influenced the rate of growth of the microalgae. A model with exponential behavior was proposed to describe the production of microalgae biomass over time. A nonlinear autoregressive model based on an Artificial Neural Network was used to predict the dynamic behavior of the $\mathrm{pH}$ during the growth of the microalgae at different light intensities. Simulations were carried out to analyze the behavior of biomass production at other light intensities within the range considered.
\end{abstract}

Keywords: Biomass of Microalgae; Haematococcus pluvialis; Modeling

\section{Introduction}

The mass cultivation of microalgae has recently aroused interest in the area of bioenergy although it has been used for many years as a source of fine chemicals, food and wastewater treatment. Currently, the photosynthesis of microalgae is considered significant for reducing emissions of carbon dioxide [1]. These microorganisms can be used to provide some different sources of renewable energy such as biodiesel, ethanol, bio-hydrogen.

The biomass can be used to produce synthesis gas, biooil by thermal treatments, or biogas by anaerobic digestion.

Microalgae also produce dyes, fatty acids, polysaccharides and vitamins that are of great interest to the pharmaceutical, food and textile sectors, among others. Thus, biodiesel from microalgae can be considered a co-product in the context of products of high added value and other commodities.

Microalgae is considered a suitable alternative for a new generation biodiesel because some species have a high oil content $[2,3]$. The possibility of energy production from biomass is promising, but with other chemicals being produced in parallel, like a bio-refinery designtype.

The use of microalgae as a feedstock for the production of biofuels has been suggested by many researchers as essential to reduce emissions of gases that cause global warming. Currently, biodiesel is produced from plants such as the soybean, sunflower, canola, cotton, castor, palm oil, among other sources, such as animal fat. However, a study reported by Crutzen et al. [4] shows that $\mathrm{N}_{2} \mathrm{O}$ emissions may cancel the beneficial effect from the use of these fuels to reduce global warming because the nitrous oxide, whose emission is accelerated due to the production method used by the agrobusiness, increases global warming. One molecule of nitrous oxide represents a potential greenhouse effect 296 times higher than one molecule of carbon dioxide [4].

The composition of microalgae in general consists of fatty acids, carotenoids, proteins, carbohydrates and minerals [5]. Haematococcus pluvialis is a green algae (Chlorophyta), mobile, single-celled, photosynthetic and capable of synthesizing and accumulating the pigment astaxanthinin response to environmental conditions [6,7]. According to Issarapayup et al. [8], this pigment has a 
higher antioxidant activity than other carotenoids, and may be used in the food industry and has great potential to fight cancer.

With the growing interest in the mass cultivation of microalgae, the kinetic modeling of the biomass production and the efficient design of photobioreactors have become important, as well as predicting performances and optimizing process operating conditions [1].

The biomass production of microalgae Haematococcus pluvialis is studied in this work. This species has a high lipid concentration and produces the pigment astaxanthin, which, due to its attractive color and biological functionality, is used as food supplement, food colorant and pigment source in aquaculture [7]. Bench scale tests were conducted to analyze the main process variables that affect the growth of microalgae: light intensity and the $\mathrm{pH}$ of the culture medium.

Biomass production was represented by an exponential model whose parameters were adjusted to describe the growth behavior of microalgae biomass over time, considering a light intensity ranging from 2000 to 10,000 lux. A nonlinear autoregressive model based on an Artificial Neural Network was also identified to describe the dynamic behavior of the $\mathrm{pH}$ during the growth of microalgae at different light intensities. Simulations were performed to predict the behavior of biomass production at other light intensities.

\section{Material and Methods}

\subsection{Microorganism Data}

The microorganism used as inoculum was the microalgae Haematococcus pluvialis. The strain is derived from the stock of microalgae Laboratory of Physiology and Cultivation of Algae, coordinated by Prof. Sergio Lourenço (Universidade Federal Fluminense, Rio de Janeiro). The microorganisms were maintained in a photoperiod chamber at a temperature of $295 \pm 1 \mathrm{~K}$ and light intensity of $35 \pm 5 \mu \mathrm{mol}$ photons $\mathrm{m}^{-2} \cdot \mathrm{s}^{-1}$.

The material was washed and sterilized at $121^{\circ} \mathrm{C}$ and 2 atm for $30 \mathrm{~min}$ in a vertical autoclave Phoenix model AV plus.

\subsection{Culture Medium}

The culture medium used was Rudic (RM) [9], adopted by Imamoglu et al. [5] to produce the microalgae biomass. The nutrient composition can be found in the above mentioned reference.

The culture medium was sterilized by autoclaving at $121^{\circ} \mathrm{C}$ and $1 \mathrm{~atm}$ for 10 minutes. The equipment used for this procedure was a vertical autoclave Phoenix model AV plus.

\subsection{Cultivation Conditions and Biomass Production Quantification}

The experiments were performed on flat plate type reactors (Figure 1), with dimensions $0.08 \times 0.2 \times 0.3 \mathrm{~m}$. The reaction volume was $3.5 \mathrm{~L}$ in which the inoculum made up $10 \%$.

The reactors were connected to an air bubble system, using an air compressor, ensuring the necessary turbulence for the mass transfer. The light intensity ranged from 2000 to 10,000 lux, the temperature was kept at $22^{\circ} \mathrm{C} \pm 1{ }^{\circ} \mathrm{C}$ and the $\mathrm{pH}$ ranged from 6.5 to 12.5 .

The reactors were inoculated inside a laminar flow chamber (Pachane, Pa 320) and then transferred to another room with controlled temperature and ambient light. The intensity of light was measured by a light meter and/or a radiometer. Both devices were calibrated in the Laboratory of Optical Properties (LAPO) (Physics Institute of Federal University of Bahia). The $\mathrm{pH}$ was measured using a $\mathrm{pH}$ meter Thermo Scientific Orion 3 Star. The calibration was performed using buffer Orion Application Solution from Thermo Scientific.

The increase in microalgal biomass was quantified using the method of counting cells in a Neubauer chamber [10] using a microscope Carl Zeiss-Axiostar Plus. The analyses were performed in duplicate.

\section{Results and Discussion}

\subsection{Model for Biomass Production in Varying Light Intensities}

The growth of biomass follows a sigmoidal curve, similar to a critically damped dynamic system of second order [11]. There is a dead time (lag phase), as can be seen by the experimental data. A model structure was proposed to represent the biomass growth (Equation (1)).

$$
\begin{cases}y(t)=y_{0}+A \cdot\left[1-\left(1+\frac{t-t_{m}}{B}\right) \cdot e^{-\frac{t-t_{m}}{B(L)}}\right] & \text { if } t>t_{m} \\ y(t)=y_{0} & \text { if } t \leq t_{m}\end{cases}
$$

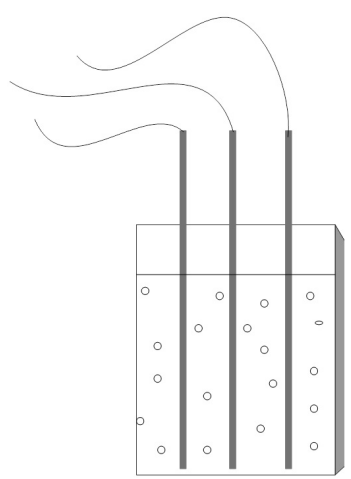

Figure 1. Reaction system: flat plate photobioreactors. 
$y$ is the microalgal biomass concentration (cells $/ \mathrm{mL}$ ), A (cells $/ \mathrm{mL}$ ) and $B(\mathrm{~h})$ are parameters of the equation, $t_{m}$ is the dead time (hours), $t$ is the time $(\mathrm{h})$ and $y_{0}$ corresponds to the initial concentration of biomass (cells $/ \mathrm{mL}$ ). The dead time or the time lag is the time associated to the adaptation of the microorganisms to the culture medium and experimental conditions.

Figures 2-4 show the experimental data and the model predictions for the light intensities of 2000, 6000 and 10,000 lux, respectively. Table 1 shows the values of the parameters ( $A$ and $B$ ) estimated in each case, considering the dead time measured directly from the experimental data. This curve fitting was performed by solving a least squares algorithm restricted to a search region (nonlinear optimization problem with inequality constraints).

The model proposed describes the growth of biomass

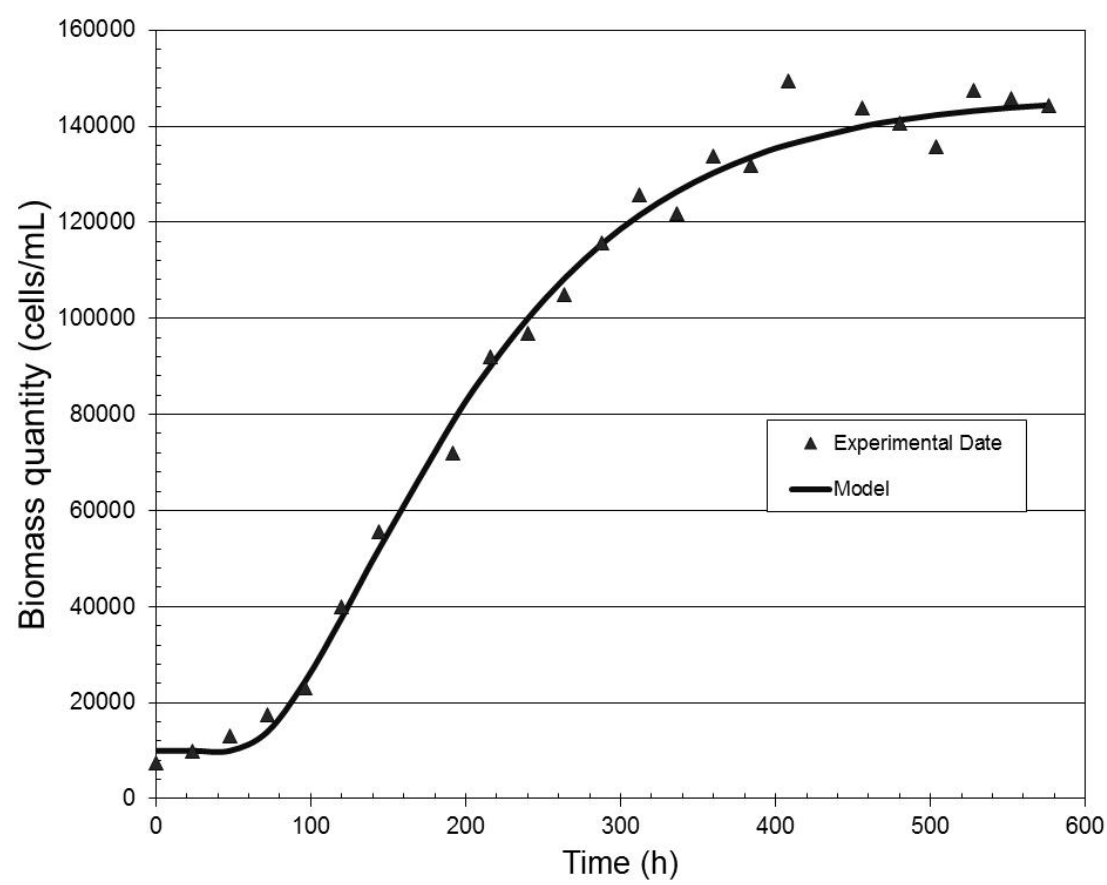

Figure 2. Biomass quantity (cells/mL) versus time (h) for the light intensity of 2000 lux.

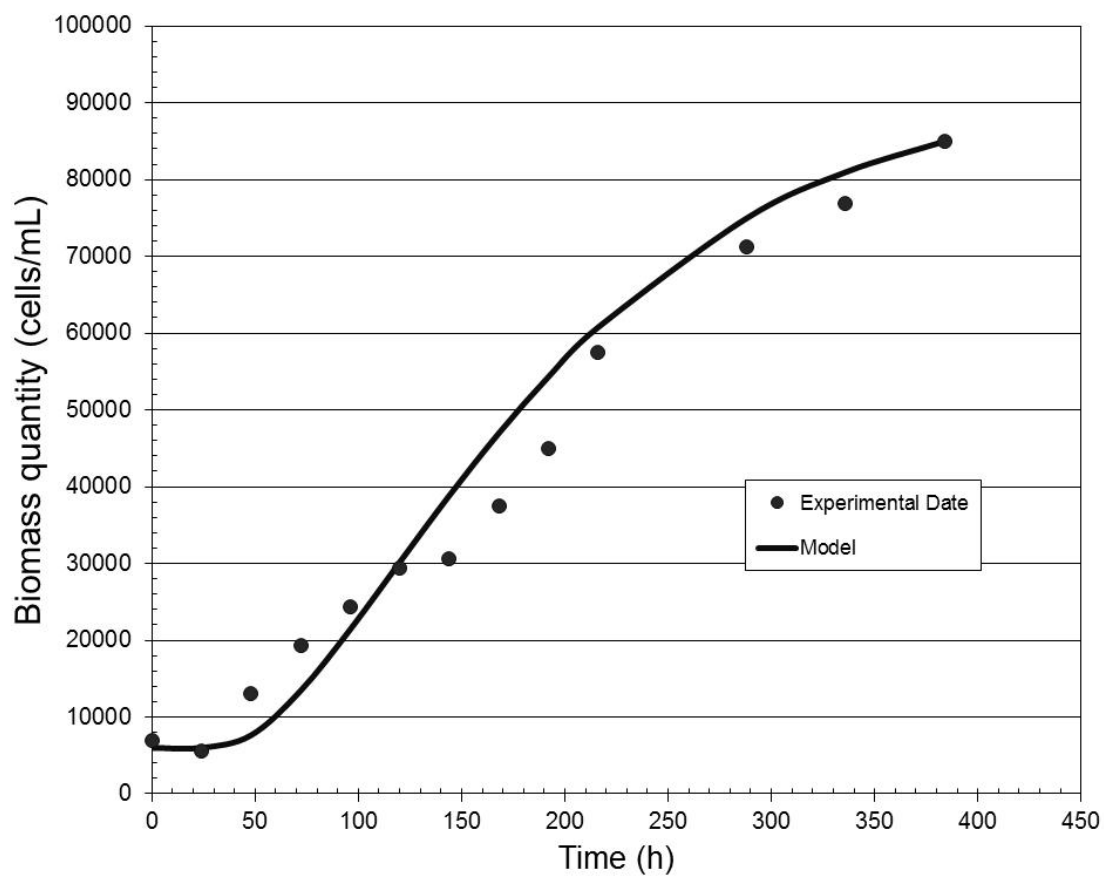

Figure 3. Biomass quantity (cells/mL) versus time (h) for the light intensity of 6000 lux. 


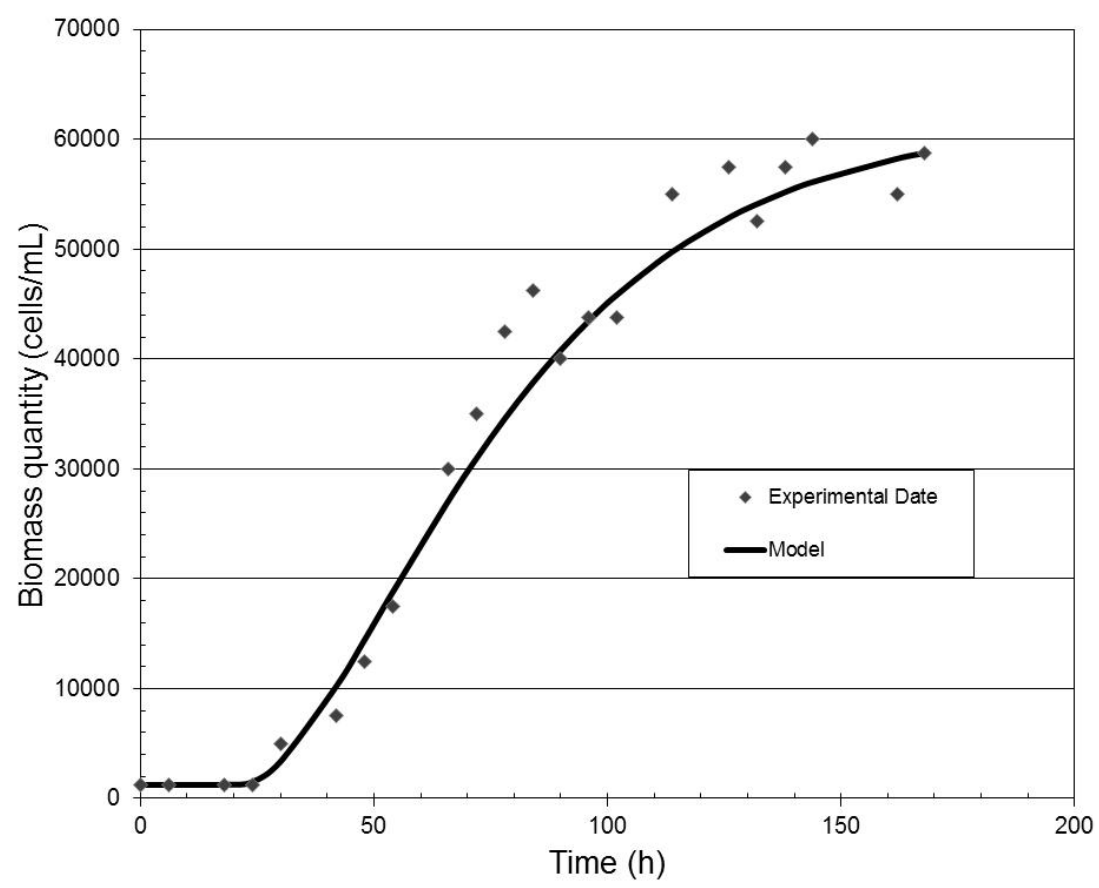

Figure 4. Biomass quantity (cells/mL) versus time (h) for the light intensity of 10,000 lux.

Table 1. Parameters of the proposed model for biomass production.

\begin{tabular}{cccc}
\hline Parameter & 2000 lux & 6000 lux & 10,000 lux \\
\hline$A($ cells $/ \mathrm{mL})$ & 136,265 & 86,261 & 60,476 \\
$B(\mathrm{~h})$ & 84 & 86 & 31 \\
$t_{m}(\mathrm{~h})$ & 50 & 30 & 21 \\
\hline
\end{tabular}

for the analyzed range of light well, with correlation coefficients equal to $0.99,0.97$ and 0.98 , respectively.

The behavior shown in the previous curves may be explained by the changes in cellular physiology. Higher light intensities produce a smaller quantity of biomass due to the trend of the cells to produce pigments, delaying growth.

Zhang et al. [12] observed that with high light intensity imposed immediately after inoculation, the culture becomes yellowish and the growth rate reduces considerably, leading to a reduction in the total biomass produced. To solve this problem the authors provided a faint light at the beginning of cultivation and then gradually increased the light intensity proportional to the increase in cell density. This strategy proved useful for both the vegetative growth of cells and for the accumulation of astaxanthin cultivation phases.

From the values of the parameters presented in Table 1, a global model was developed in order to predict the production of biomass from Haematococcus pluvialis microalgae at any light intensity between the range of 2000 to 10,000 lux. This model was generated by adjusting the parameters as a function of light intensity, according to a polynomial function (Equation (2)).

$$
\left\{\begin{array}{l}
y(t)=y_{0}+A(L) \cdot\left[1-\left(1+\frac{t-t_{m}(L)}{B(L)}\right) \cdot e^{-\frac{t-t_{m}(L)}{B(L)}}\right] \\
\text { if } t>t_{m}(L) \\
y(t)=y_{0} \quad \text { if } t \leq t_{m}(L)
\end{array}\right.
$$

where $L$ is the light intensity (lux).

Figures 5 and $\mathbf{6}$ show the theoretical curves for biomass production using this model, in the range of 3000 to 9000 lux. A reduction in biomass production at the end of the batch is observed for light intensities ranging from 3000 to 5000 lux. For light intensities between 7000 9000 lux there is an increase in the growth rate at the initial stage, but a reduction in the amount of biomass at the final stage of the process.

Results described by Boussiba and Vonshak [13] and Zhang et al. [12] show that the increase in the accumulation of astaxanthin at high light intensities, caused by changes in cellular metabolism, causes a change in the growth rate of microalgae production leading to a lower production of the total biomass, according to the results showed by the proposed model.

Low light intensities are usually insufficient for the accumulation of astaxanthin, as noted by DominguezBocanegra et al. [14]. Therefore it is important to use an adequate light intensity to prioritize both the vegetative growth of the cells and the gradual accumulation of astaxanthin within the cells. 


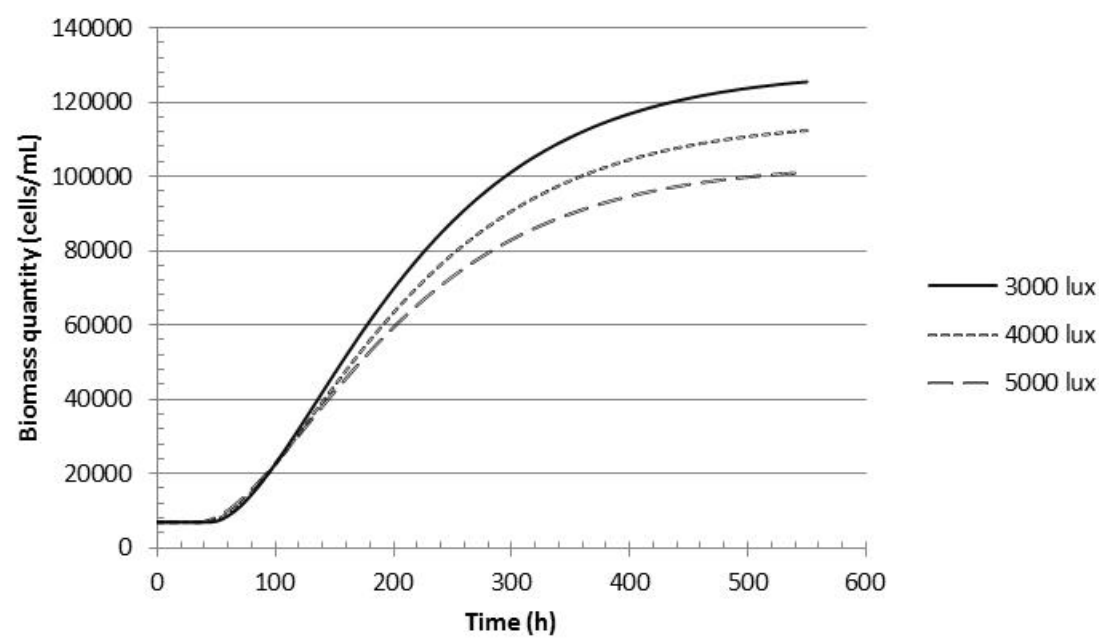

Figure 5. Biomass quantity (cells/mL) versus time (h) for the light intensities of 3000, 4000 and 5000 lux.

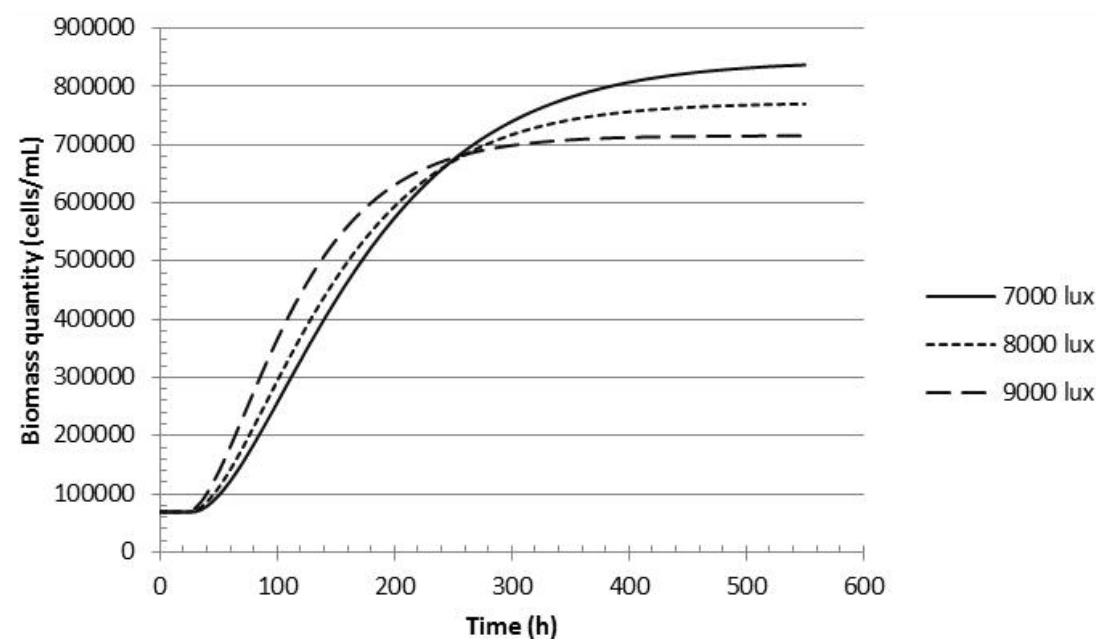

Figure 6. Biomass quantity (cells/mL) versus time (h) for the light intensities of 7000, 8000 and 9000 lux.

\subsection{Autoregressive Model for the Prediction of Dynamic Behavior of pH at Different Light Intensities}

The $\mathrm{pH}$ of the culture medium follows a nonlinear behavior. A nonlinear autoregressive model based on an Artificial Neural Network (ANN) was used to predict this behavior at different light intensities.

The configuration that provided the best results comprised an ANN with two neurons in the hidden layer considering only one past value of $\mathrm{pH}$ in the input layer. Figure 7 shows this structure which has the ability to predict, recursively, the changes in $\mathrm{pH}$ over time. The activation functions adopted in the hidden neurons and in the output neuron were sigmoid and linear functions, respectively.

Seven parameters were estimated for each light intensity (four synaptic weights and three biases, namely, $w_{1}$, $w_{2}, w_{3}, w_{4}, b_{1}, b_{2}$ and $\left.b_{3}\right)$.

The $\mathrm{pH}$ value predicted by the ANN is given by Equa- tion (3):

$$
\mathrm{pH}(t+1)=y_{1} \cdot w_{3}+y_{2} \cdot w_{4}+b_{3}
$$

where:

$$
y_{1}=\frac{1}{1+\mathrm{e}^{-\left[p H(t) \cdot w_{1}+b_{1}\right]}}
$$

and

$$
y_{2}=\frac{1}{1+\mathrm{e}^{-\left[p H(t) \cdot w_{2}+b_{2}\right]}}
$$

The parameters were adjusted for each light intensity in order to obtain a global model to predict the $\mathrm{pH}$ in the range of 2000 to 10,000 lux.

Figure 8 show the $\mathrm{pH}$ curves versus time using ANN and the experimental data for light intensities of 2000, 6000 and 10,000 lux respectively. The correlation coefficients calculated after the adjustment of the $\mathrm{pH}$ curves for 2000, 6000 and 10,000 lux were 0.9076, 0.9995 and 


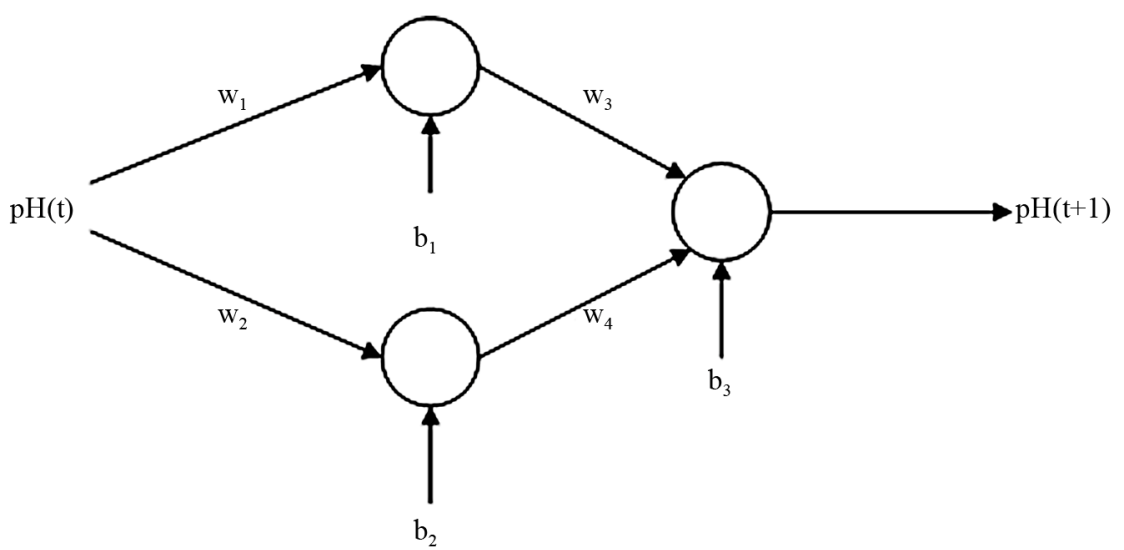

Figure 7. System proposed for the artificial neural network.

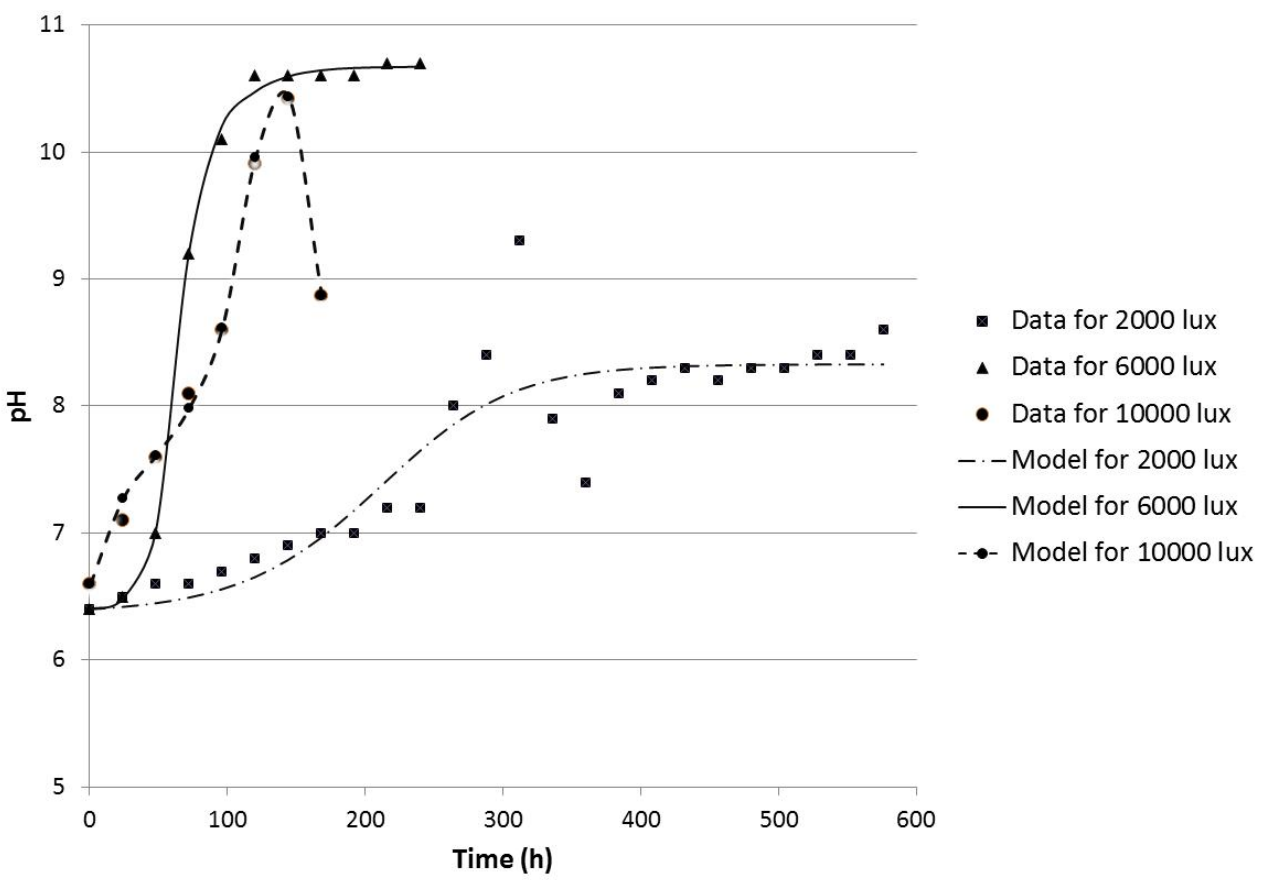

Figure 8. Behavior of $\mathrm{pH}$ versus time in the range of 2000 to 10,000 lux.

0.9982 respectively.

The greater the light intensity in the medium, the sooner the $\mathrm{pH}$ increases, thus discouraging microbial growth and promoting the production of astaxanthin. This explains the higher specific growth rate observed for the 2000 lux experiments when compared to those with 10,000 lux.

In the photosynthesis reaction (Equation (6)) [15], the use of carbon dioxide results in a shift in the balance of the dissolution reaction of this gas in water (Equation (7)), favoring a reduction in protons $\mathrm{H}^{+}$in the medium. Thus, a higher light intensity favors the photosynthesis process, increasing the $\mathrm{pH}$, a fact observed during all the batch experiments without $\mathrm{pH}$ control.

$$
6 \mathrm{CO}_{2}+6 \mathrm{H}_{2} \mathrm{O} \rightarrow \mathrm{C}_{6} \mathrm{H}_{12} \mathrm{O}_{6}+6 \mathrm{O}_{2}
$$

$$
\mathrm{CO}_{2(\mathrm{~g})}+\mathrm{H}_{2} \mathrm{O} \rightleftharpoons \mathrm{HCO}_{3(\mathrm{aq})}^{-}+\mathrm{H}_{\mathrm{aq}}^{+} \rightleftharpoons \mathrm{CO}_{3(\mathrm{aq})}^{2-}+2 \mathrm{H}_{\mathrm{aq}}^{+}
$$

High light intensities can generate lower yields of biomass as a result of the increase in $\mathrm{pH}$. Thus, to obtain an appropriate batch culture without $\mathrm{pH}$ control, if the goal is vegetative growth, the use of light intensity that varies from 2000 to 6000 lux is recommended for the cultivation of Haematococcus pluvialis microalgae. If the goal is astaxanthin production, the increase in light intensity must be performed gradually to avoid a significant decrease in productivity. The evaluation and control of $\mathrm{pH}$ in the culture medium is of great importance because the concentration of hydrogen ions is one of the most important factors that can affect the growth of algae cells [16]. The concentration of hydrogen ions influences the metabolism of the microorganisms and thus deter- 
mines the biosynthesis of secondary metabolites such as the astaxanthin product.

\section{Conclusions}

An empirical model for predicting the production of biomass of the microalgae Haematococcus pluvialis with light intensities ranging from 2000 to 10,000 lux is presented and analysed. The behavior of the $\mathrm{pH}$ of the culture media is also predicted using a model based on an artificial neural network (ANN) for the same range of light intensities. Both models described the expected experimental behavior reasonably well.

Although it is expected that an increase in light intensity helps accelerate the rate of growth of microalgae biomass, it also causes a reduction in the amount of Haematococcus pluvialis biomass produced due to the increase in the accumulation of the pigment astaxanthin. The greater the light intensity in the culture medium, the sooner the $\mathrm{pH}$ increases, influencing the cell metabolism inhibiting microbial growth and promoting the production of astaxanthin.

For successful batch cultivation of Haematococcus pluvialis microalgae without $\mathrm{pH}$ control, the use of a light intensity that varies from 2000 to 6000 lux is recommended if the goal is vegetative growth. On the other hand, if the goal is to produce astaxanthin, there must be a gradual increase in light intensity to avoid a significant decrease in the biomass yield.

\section{Acknowledgements}

The authors express their gratitude to the Universidade Federal da Bahia, CNPq and CAPES.

\section{REFERENCES}

[1] Y. S. Yun and J. M. Park, "Kinetic Modeling of the Light-Dependent Photosynthetic Activity of the Green Microalga Chlorella vulgaris," Biotechnology and Bioengineering, Vol. 83, No. 3, 2003, pp. 303-311. doi:10.1002/bit.10669

[2] S. Amin, "Review on Biofuel Oil and Gas Production Processes from Microalgae," Energy Conversion and Management, Vol. 50, No. 7, 2009, pp. 1834-1840. doi:10.1016/j.enconman.2009.03.001

[3] L. Gouveia and A. C.Oliveira, "Microalgae as a Raw Material for Biofuels Production," Journal of Industrial Microbiology \& Biotechnology, Vol. 36, No. 2, 2009, pp. 269-274. doi:10.1007/s10295-008-0495-6

[4] P. J. Crutzen, et al., " $\mathrm{N}_{2} \mathrm{O}$ Release from Agro-Biofuel
Production Negates Global Warming Reduction by Replacing Fossil Fuels," Atmospheric Chemistry and Physics, Vol. 8, No. 2, 2008, pp. 389-395. doi:10.5194/acp-8-389-2008

[5] E. Imamoglu, F. V. Sukan and M. C. Dalay, "Effect of Different Culture Media and Light Intensities on Growth of Haematococcus pluvialis," International Journal of Natural and Engineering Sciences, Vol. 1, No. 3, 2007, pp. 5-9.

[6] Q. L. Dong and X. M. Zhao, "In Situ Carbon Dioxide Fixation in the Process of Natural Astaxanthin Production by a Mixed Culture of Haematococcus pluvialis and Phaffiarhodozyma," Catalysis Today, Vol. 98, No. 4, 2004, pp. 537-544. doi:10.1016/j.cattod.2004.09.052

[7] V. Ghiggi, "Estudo do Crescimento e Indução da Produção do pigmentoastaxantinapor Haematococcus pluvialis, Dissertação (Mestrado)," Programa de Pós-Graduaçãoem Engenharia de Bioprocessos e Biotecnologia, Universidade Federal do Paraná, Curitiba, 2007.

[8] K. Issarapayup, S. Powtongsook and P. Pavasant, "Flat Panel Airlift Photobioreactors for Cultivation of Vegetative Cells of Microalga Haematococcus pluvialis," Journal of Biotechnology, Vol. 142, No. 3-4, 2009, pp. 227 232. doi:10.1016/j.jbiotec.2009.04.014

[9] V. Rudic and T. Dudnícenco, "Process for Cultivation of Green Alga Haematococcus pluvialis (Flotow)," MD Patent Nr. A 2000 0154, 2000.

[10] S. O. Lourenço, "Cultivo de Microalgas Marinhas: Princípios e Aplicações,” Rima, São Carlos, 2006.

[11] G. Sthephanopoulos, "Chemical Process Control," Prentice-Hall, Upper Saddle River, 1984.

[12] B. Y. Zhang, et al., "Production of Astaxanthin from Haematococcus in Open Pond by Two-Stage Growth One-Step Process," Aquaculture, Vol. 295, No. 3-4, 2009, pp. 275-281. doi:10.1016/j.aquaculture.2009.06.043

[13] S. Boussiba and A. Vonshak, "Astaxanthin Accumulation in the Green Algae Haematococcus pluvialis," Plant Cell Physio, Vol. 32, No. 7, 1991, pp. 1077-1082.

[14] A. R. Dominguez-Bocanegra, T. Ponce-Noyola and J. A. Torres-Munoz, "Astaxanthin Production by Phaffiarhodozyma and Haematococcus pluvialis: A Comparative Study," Applied Microbiology and Biotechnology, Vol. 75, No. 4, 2007, pp. 783-791. doi:10.1007/s00253-007-0889-9

[15] L. Taiz and E. Zeiger, "Fisiologia Vegetal," 3rd Edition, Artmed, Porto Alegre, 2004.

[16] Z. I. Khalil, et al., "Effect of pH on Growth and Biochemical Responses of Dunaliellabardawil and Chlorella ellipsoidea," World Journal of Microbiology \& Biotechnology, Vol. 26, No. 7, 2010, pp. 1225-1231. doi:10.1007/s11274-009-0292-Z 